\title{
Análise da resistência em concreto reforçado com fibras de aço, através de banco de dados
}

O concreto reforçado com fibra de aço (CRFA) é um importante elemento da engenharia civil a ser estudado, apesar de o mesmo estar presente na construção civi desde a década de 1960, ainda é pouco utilizado no Brasil. Com a crescente aplicabilidade do CRFA, os códigos de projeto têm se empenhado em melhorar a caracterização dos materiais utilizados para que haja melhor confiança nos comportamentos das peças, minimizando as disparidades entre os resultados obtidos em laboratório com a realidade na obra. Este artigo tem como objetivo explicar o desenvolvimento da caracterização do CRFA no estado endurecido, referente ao ensaio de três pontos e analisar a confiabilidade da recomendação normativa RILEM TC 162-TDF (2002), através de um banco de dados composto por 68 especimes. A partir de análises estatísticas do banco de dados que reúne resultados experimentais sobre flexão de vigas com adição de fibra e armadura convencional, verificou-se que $22,05 \%$ dos valores deram segundo a classificação da escala de demérito em 'Perigosa'. Conclui-se que confiabilidade para alguns tipos de vigas utilizando os devidos materiais é atendida pela RILEM, porém quando utilizado o concreto de alto desempenho os resultados tendem a ficarem mais dispersos e inseguros.

Palavras-chave: Concreto Armado; Flexão; Concreto Reforçado com Fibra de Aço.

\section{Strength analysis of steel fiber reinforced concrete by database}

The steel fibre reinforced concrete (SFRC) is an important element of civil engineering to be studied, although it has been present in civil construction since the 1960 s, it is still little used in Brazil. With the increasing applicability of the SRFC, the project codes have been focused on improving the characterization of the materials used so that there is better confidence in the behavior of the parts, minimizing the disparities between the results obtained in the laboratory and the reality in the work. This paper aims to explain or develop the characterization of long-term CRFA for three-point assays and to analyze the recommendations of normative recommendations RILEM TC 162-TDF (2002), using a database of 68 species. From statistical data from the database that gathers experimental results on bending of beams with addition of fiber and conventional reinforcement, it was found that $22.05 \%$ of the values given according to the demerit scale classification as 'Dangerous'. It turns out that we use for some types of vibrations that use the proper materials is hosted by RILEM, but when we use high performance concrete to get more dispersed and unsafe results.

Keywords: Reinforced Concrete; Flexural; Steel fibre reinforced concrete.

\section{Topic: Engenharia de Estruturas}

Reviewed anonymously in the process of blind peer.
Received: $12 / 08 / 2019$

Approved: $22 / 11 / 2019$
Bruna de Sousa Franca

Instituto Tocantinense Presidente Antônio Carlos, Brasil http://lattes.cnpq.br/4836793820475497

bsfranca4@gmail.com

Douglas Freitas Augusto dos Santos

Instituto Tocantinense Presidente Antônio Carlos, Brasil

http://lattes.cnpq.br/6181125172616043

eng.prof.santos@gmail.com

\section{Referencing this:}

FRANCA, B. S.; SANTOS, D. F. A.. Análise da resistência em concreto reforçado com fibras de aço, através de banco de dados. Engineering Sciences, v.7, n.3, p.64-72, 2019. DOI:

http://doi.org/10.6008/CBPC2318-3055.2019.003.0006 


\section{INTRODUÇÃO}

O concreto é um dos elementos da construção civil mais utilizados na atualidade, composto por cimento, agregados e água ele também pode conter aditivos ou não. Existem várias vantagens pelo qual o concreto é tão popular, uma delas é a capacidade que o construtor tem de executar diversos formatos e ter uma liberdade de trabalho maior.

Porém o concreto convencional possui algumas limitações. Em um concreto convencional, uma fissura irá proporcionar uma barreira a propagação de tensões de tração. Quando ocorre uma fissura essa tensão tende a se acumular nas extremidades da fissura. E se as tensões expostas superarem a tensão crítica, ocorrerá uma ruptura abrupta do material, no caso que esse concreto esteja exposto a tensões cíclicas, pode interpretar da mesma maneira. Assim, como em cada ciclo há uma propagação de microfissuras, acontece a propagação e a ruptura do material por fadiga (Figura 1a), quando o concreto convencional é rompido ele perde sua capacidade resistente tração, tendo um comportamento tipicamente frágil. Quando as fibras de aço são inseridas no concreto, elas atuam como ponte de transmissão das tensões, interceptando as fissuras e atuando como obstáculo de propagação as microfissuras (Figura 1b), o concreto consequentemente passa a ter um comportamento pseudo-ductil ou não frágil, possuindo capacidade de resistência pós-fissuração (VELASCO, 2009).

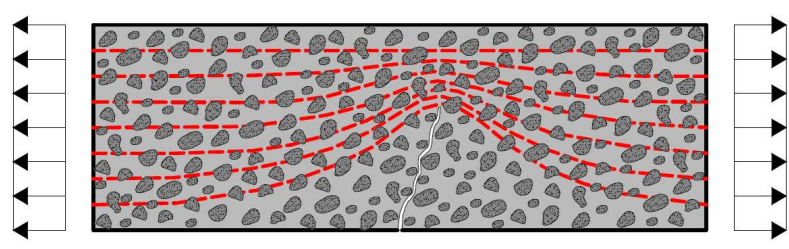

a) Distribuição das tensões no concreto simples fissurado.

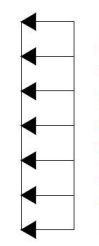

b) Distribuiç̧ão das tensões no CRFA fissurado.

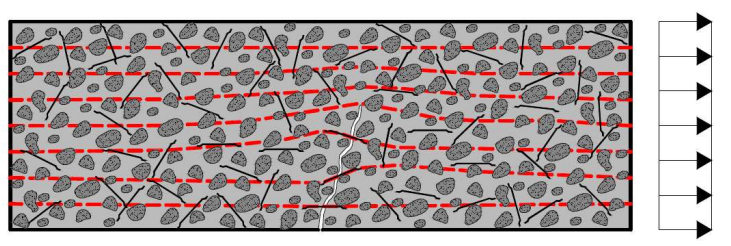

Figura 1: Distribuição das tensões (linhas vermelhas) no concreto.

Segundo Velasco (2009), o comportamento do CRFA de tração na flexão é o mais importante do estudo, a aplicação real do concreto, levando em conta que, na maioria das aplicações a fibra está sujeita a esse esforço. Além disso, esse é o aspecto do qual a fibra apresenta maiores ganhos em relação a outros tipos de concreto. Após o surgimento da primeira fissura, a carga de pico pode ser equivalente ao dobro ou triplo da carga de primeira fissura (Figura 2). Esse comportamento depende do volume de fibra e do tipo utilizado. $O$ ensaio de tração na flexão irá seguir uma determinada norma de acordo com a escolha do autor, nesse caso foram usadas a norma RILEM TC 162-TDF (2002).

Essa pesquisa é importante pois contribui para aumentar do conhecimento sobre o comportamento de elementos estruturais de CRFA e sobre as variáveis atuantes. Devido à escassez de recomendações normativas e a grande variedade de hipóteses de comportamento assumidas por diversos pesquisadores. $O$ presente trabalho tem como objetivo avaliar o método proposto pelo RILEM (2002) através da norma TC 162-TDF (2003) de estimativa do momento resistente de vigas de CRFA, o método é testado avaliando as estimativas do momento resistente máximo para 62 vigas de CRFA comparadas aos valores obtidos experimentalmente. Com base nesta análise, são determinados os níveis de segurança associados à utilização 
do método para o dimensionamento de vigas de CRFA. Nas estimativas segundo as recomendações do RILEM, utilizou-se para determinação dos parâmetros de tensão residual do CRFA as formulações empíricas apresentadas no relatório anterior esta disciplina.

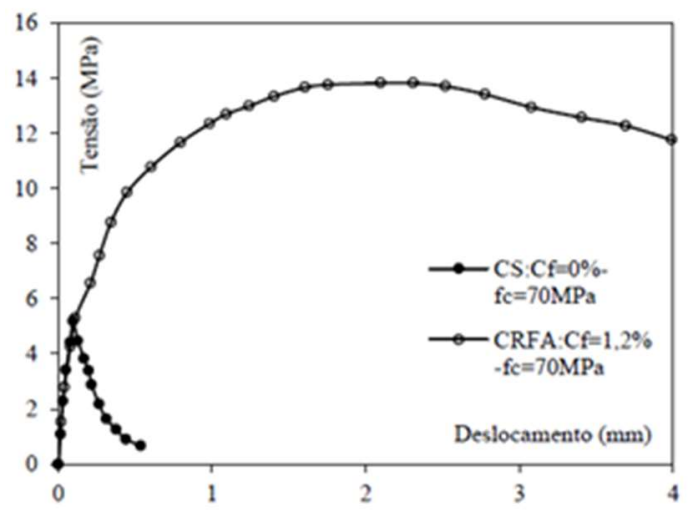

Figura 2: Relação tensão-deformação.

\section{METODOLOGIA}

O quadro abaixo resume as variáveis consideradas em 14 estudos, realizados entre os anos de 1981 à 2014, sobre comportamento à flexão de vigas de concreto com armadura convencional e fibras, totalizado 68 espécimes. Nela constata-se que as dimensões das seções transversais adotadas são geralmente pequenas, com a altura $(h)$ e base $(b)$ variando entre 300 a $100 \mathrm{~mm}$ respectivamente; um dos parâmetros mais relevantes é o volume de fibras ( $V f$ ), que teve teor máximo de 2,0\% e mínimo de 0,25\%. Quanto a taxa de armadura, em quatro estudos a taxa de armadura de compressão foi totalmente retirada, nos estudos do Naghibdehi (2014) não há armadura de compressão. O valor da taxa de armadura longitudinal de tração foi maior que $1 \%$ em 25 vigas.

Os estudos de Lima (2012) e Rodrigues Jr. (2009), se concentraram em investigar a influência da adição de fibras em vigas, realizando uma redução na armadura convencional por fibras de aço, Fritih (2012), se fez estudo com CRFA analisando o comportamento a flexão e cisalhamento, Barbos (2013), Khalil (2013) e Beshara (2012), assim como os autores já citados, fizeram estudos voltados para medir a resistência a flexão de vigas com armaduras convencionais e reforço de fibras de aço, contudo, trata-se de concreto de alto desempenho (elevados fc). Uma análise de CRFA em vigas caixa foi realizada em 2005. O Quadro 1 apresenta o banco de dados elaborado a partir da literatura cientifica.

Quadro 1: Banco de Dados.

\begin{tabular}{|c|c|c|c|c|c|c|c|c|}
\hline Autor & Peça & $B(\mathrm{~mm})$ & $D(\mathrm{~mm})$ & $V f(\%)$ & If $/ d f$ & $F c(M P a)$ & $P(\%)$ & $M_{R, \operatorname{EXP}}(k N . m)$ \\
\hline \multirow{4}{*}{ LIMA (2012) } & Vigas 6 & 150 & 205 & 0,5 & 80,0 & 62,80 & 0,49 & 19,34 \\
\hline & Vigas 7 & 150 & 205 & 0,5 & 80,0 & 62,80 & 0,49 & 11,80 \\
\hline & Vigas 10 & 150 & 205 & 0,3 & 80,0 & 63,30 & 0,28 & 12,60 \\
\hline & Vigas 13 & 150 & 205 & 0,8 & 80,0 & 63,00 & 0,28 & 11,03 \\
\hline \multirow{6}{*}{ GRIBNIAK et al. (2012) } & S3 -1 - F05 & 150 & 278 & 0,5 & 53,0 & 55,00 & 0,57 & 44,50 \\
\hline & S3 -2 - F05 & 150 & 278 & 0,5 & 53,0 & 55,00 & 0,57 & 46,00 \\
\hline & S3 -1 - F10 & 150 & 278 & 1,0 & 53,0 & 48,00 & 0,57 & 52,00 \\
\hline & S3 -2 - F10 & 150 & 278 & 1,0 & 53,0 & 48,00 & 0,57 & 52,30 \\
\hline & S3 -1 - F15 & 150 & 278 & 1,5 & 53,0 & 52,00 & 0,57 & 54,00 \\
\hline & S3 -2 - F15 & 150 & 278 & 1,5 & 53,0 & 52,00 & 0,57 & 53,60 \\
\hline FRITIH (2012) & Serie 'A' FRSCC & 150 & 253 & 0,3 & 18,8 & 50,00 & 0,81 & 18,93 \\
\hline
\end{tabular}




\begin{tabular}{|c|c|c|c|c|c|c|c|c|}
\hline ALTUN (2005) & S01 - 0,4 & 300 & 278 & 0,4 & 79,8 & 122,00 & 0,41 & 60,30 \\
\hline \multirow{22}{*}{ BESHARA (2012) } & Serie [6] - 0,5 & 120 & 155 & 0,5 & 50,0 & 80,00 & 0,84 & 12,58 \\
\hline & Serie [6] - 1,0 & 120 & 155 & 1,0 & 50,0 & 85,00 & 0,84 & 13,55 \\
\hline & Serie [6] - 2,0 & 120 & 155 & 2,0 & 50,0 & 89,00 & 0,84 & 14,02 \\
\hline & Serie [13] - 0,75 & 100 & 130 & 0,8 & 75,0 & 85,00 & 1,21 & 9,60 \\
\hline & Serie [13] - 1,50 & 100 & 130 & 1,5 & 75,0 & 85,00 & 1,21 & 10,95 \\
\hline & Serie [10] - 0,50 & 100 & 80 & 0,5 & 30,0 & 80,00 & 1,96 & 4,85 \\
\hline & Serie [10] - 1,00 & 100 & 80 & 1,0 & 50,0 & 80,00 & 1,96 & 5,00 \\
\hline & Serie [10] - 1,50 & 100 & 80 & 1,5 & 30,0 & 80,00 & 1,96 & 5,40 \\
\hline & Serie [14] - 0,5 & 100 & 105 & 0,5 & 50,0 & 42,00 & 1,50 & 6,87 \\
\hline & Serie $[14]-1,0$ & 100 & 105 & 1,0 & 50,0 & 42,00 & 1,50 & 7,19 \\
\hline & Serie [14] - 1,5 & 100 & 105 & 1,5 & 50,0 & 42,00 & 1,50 & 7,22 \\
\hline & $:>$ & 100 & 105 & 2,0 & 50,0 & 42,00 & 1,50 & 7,51 \\
\hline & Serie [15] - 0,5 & 250 & 228 & 0,5 & 75,0 & 35,00 & 0,70 & 61,20 \\
\hline & Serie [15] - 1,0 & 250 & 228 & 1,0 & 60,0 & 35,00 & 0,70 & 61,50 \\
\hline & Serie [15] - 1,5 & 250 & 228 & 1,5 & 75,0 & 35,00 & 0,70 & 64,80 \\
\hline & Serie [16] - 0,5 & 170 & 280 & 0,5 & 75,0 & 87,00 & 1,32 & 90,69 \\
\hline & Serie [16] - 1,0 & 170 & 278 & 1,0 & 75,0 & 88,00 & 1,33 & 95,41 \\
\hline & Serie $[16]-1,5$ & 170 & 278 & 1,5 & 75,0 & 90,00 & 1,33 & 100,40 \\
\hline & Serie [17] - 1,2 & 100 & 120 & 1,2 & 60,0 & 46,00 & 0,21 & 3,12 \\
\hline & Serie [17] - 1,2 & 100 & 120 & 1,2 & 60,0 & 46,00 & 2,54 & 17,40 \\
\hline & Serie [17] - 0,89 & 100 & 120 & 0,9 & 75,0 & 42,00 & 0,21 & 2,90 \\
\hline & Serie [17] - 0,89 & 100 & 120 & 0,9 & 75,0 & 42,00 & 2,54 & 17,27 \\
\hline \multirow{2}{*}{ BĂRBOS (2013) } & FT 5.1-1 & 130 & 223 & 0,4 & 62,5 & 54,00 & 1,76 & 50,00 \\
\hline & FT 5.2-1 & 130 & 223 & 0,4 & 62,5 & 66,00 & 1,76 & 50,00 \\
\hline \multirow{6}{*}{ KANG et al. (2012) } & FHC-65-1.5 & 125 & 210 & 0,5 & 67,0 & 63,90 & 1,53 & 45,73 \\
\hline & FHC-100-1.5 & 125 & 210 & 0,8 & 67,0 & 68,60 & 1,53 & 48,93 \\
\hline & FNC-65-1 & 125 & 210 & 1,0 & 67,0 & 30,80 & 1,01 & 37,11 \\
\hline & FNC-100-1 & 125 & 210 & 1,3 & 67,0 & 32,60 & 1,01 & 37,86 \\
\hline & FNC-65-1.5 & 125 & 210 & 1,6 & 67,0 & 30,80 & 1,53 & 41,88 \\
\hline & FNC-100-1.5 & 125 & 210 & 1,8 & 67,0 & 32,60 & 1,53 & 43,11 \\
\hline \multirow{3}{*}{ KHALIL (2013) } & D250V0,5h & 150 & 200 & 0,5 & 50,0 & 60,00 & 0,75 & 25,20 \\
\hline & D250V0,75h & 150 & 200 & 0,8 & 50,0 & 60,00 & 0,75 & 27,60 \\
\hline & D250V1,0h & 150 & 200 & 1,0 & 50,0 & 60,00 & 0,75 & 29,10 \\
\hline \multirow{7}{*}{ RODRIGUEZ JR (2009) } & VIGA 1-1 & 150 & 240 & 0,4 & 75,0 & 42,00 & 0,26 & 20,70 \\
\hline & VIGA 1-2 & 150 & 240 & 0,8 & 75,0 & 53,00 & 0,26 & 25,28 \\
\hline & VIGA 1-3 & 150 & 240 & 1,2 & 75,0 & 45,60 & 0,26 & 27,83 \\
\hline & \multicolumn{8}{|c|}{ Continuação Quadro 2: Banco de Dados Continuação } \\
\hline & VIGA 2-1 & 150 & 240 & 0,4 & 75,0 & 47,80 & 0,56 & 39,00 \\
\hline & VIGA 2-2 & 150 & 240 & 0,8 & 75,0 & 53,00 & 0,56 & 46,35 \\
\hline & VIGA 2-3 & 150 & 240 & 1,2 & 75,0 & 45,60 & 0,56 & 42,00 \\
\hline \multirow{6}{*}{ SWAMY et al. (1981) } & DR11 & 130 & 176 & 0,5 & 100,0 & 30,79 & 0,99 & 23,25 \\
\hline & DR12 & 130 & 176 & 1,0 & 100,0 & 31,20 & 0,99 & 23,81 \\
\hline & DR21 & 130 & 176 & 0,5 & 100,0 & 29,09 & 1,76 & 33,68 \\
\hline & DR22 & 130 & 176 & 1,0 & 100,0 & 31,35 & 1,76 & 35,06 \\
\hline & DR31 & 130 & 176 & 0,5 & 100,0 & 30,91 & 0,99 & 28,62 \\
\hline & DR32 & 130 & 176 & 1,0 & 100,0 & 31,88 & 0,99 & 30,83 \\
\hline \multirow{4}{*}{ BYUNG (1992) } & S1V1 & 120 & 140 & 1,0 & 57,0 & 43,00 & 1,51 & 15,23 \\
\hline & S1V2 & 120 & 140 & 2,0 & 57,0 & 47,80 & 1,51 & 17,96 \\
\hline & S2V1 & 120 & 140 & 1,0 & 57,0 & 43,00 & 2,36 & 22,64 \\
\hline & S2V2 & 120 & 140 & 2,0 & 57,0 & 47,80 & 2,36 & 23,37 \\
\hline LOPES (2005) & $\mathrm{V} 2$ & 125 & 228 & 2,0 & 64,0 & 64,22 & 1,41 & 55,77 \\
\hline \multirow{4}{*}{ KOVÁCS et al. (2003) } & A5 & 100 & 120 & 0,5 & 30,0 & 39,85 & 3,35 & 21,06 \\
\hline & A6 & 100 & 120 & 1,0 & 30,0 & 38,55 & 3,35 & 21,00 \\
\hline & A8 & 100 & 120 & 0,5 & 30,0 & 39,85 & 3,35 & 21,00 \\
\hline & A9 & 100 & 120 & 1,0 & 30,0 & 38,55 & 3,35 & 21,96 \\
\hline \multirow{2}{*}{ NAGHIBDEHI et al. (2014) } & PP0.5 & 100 & 270 & 0,5 & 47,6 & 49,36 & 0,00 & 3,20 \\
\hline & PP1.0 & 100 & 270 & 1,0 & 47,6 & 55,50 & 0,00 & 4,68 \\
\hline
\end{tabular}

Metodologia de Cálculo

O RILEM TC 162-TDF (2002) estabelece que o comportamento do CRFA à tração seja avaliado em função das áreas sob a curva carga x deslocamento (Figuraa), ou em função da curva carga x CMOD (Crack 
Mouth Opening Displacement), CMOD (Figurab), as quais são obtidas do teste de flexão em três pontos em vigas entalhadas (Figura ). O procedimento do RILEM estabelece o limite de proporcionalidade do material, fct,L, ver a equação Equação 1, as resistências equivalentes à tração na flexão, feq,j (j=2 e 3) , ver a Equação 2, e as resistências residuais à tração na flexão, $f_{R, i}(i=1,2,3$ e 4), ver a equação Equação 3, Se o objetivo do teste for o cálculo das resistências equivalentes à tração na flexão, é necessário realizar a medida apenas do deslocamento, porém, se a intenção for determinar os valores das resistências residuais à tração na flexão, é possível optar pela medida do deslocamento ou do CMOD.

$$
\begin{gathered}
f_{f c t, L}=\frac{3 \cdot F_{L} \cdot L}{2 \cdot b \cdot h_{s p}{ }^{2}} \\
f_{e q, 2}=\frac{3}{2} \cdot \frac{D_{B Z, 2}^{f} \cdot \frac{L}{0.50} \cdot \frac{L}{b \cdot h_{s p}{ }^{2}}}{f_{R, i}=\frac{3 \cdot F_{R, i} \cdot L}{2 \cdot b \cdot h_{s p}^{2}}} \\
f_{e q, 3}=\frac{3}{2} \cdot \frac{D_{B Z, 3}^{f}}{2.50} \cdot \frac{L}{b \cdot h_{s p}{ }^{2}}
\end{gathered}
$$

Equação 1

Equação 2

Equação 3

Equação 4

$f c t, L$ : resistência no limite de proporcionalidade do material ( $\mathrm{MPa}$ ) $f e q, j$ = Resistências equivalentes à tração na flexão (MPa); $f R, i=$ Resistências residuais à tração na flexão (MPa); $\mathrm{b}=$ largura do corpo de prova $(\mathrm{mm})$;

$h_{s p}=$ distância entre a extremidade do entalhe e o topo da seção transversal $(\mathrm{mm})$ $\mathrm{L}=$ vão de ensaio $(\mathrm{mm})$ $\mathrm{F}_{\mathrm{L}}$ : carga no limite de proporcionalidade $(\mathrm{KN})$ $D^{f}{ }_{B z}=$ área de energia absorvida até deslocamento (N.mm)



Figura 3: Ensaio de flexão em três pontos em viga entalhada (dimensões em mm).

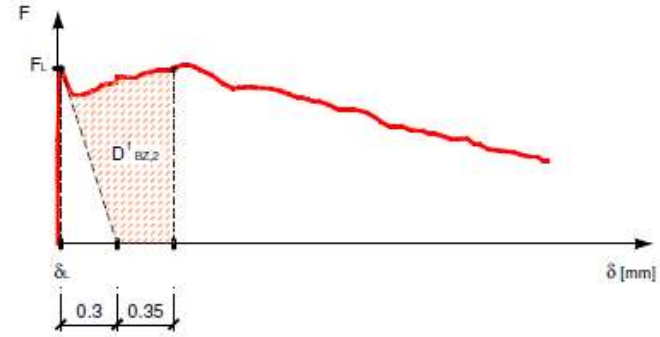

$\left.a_{1}\right)$ Avaliação de $f_{\text {eq,2 }}$

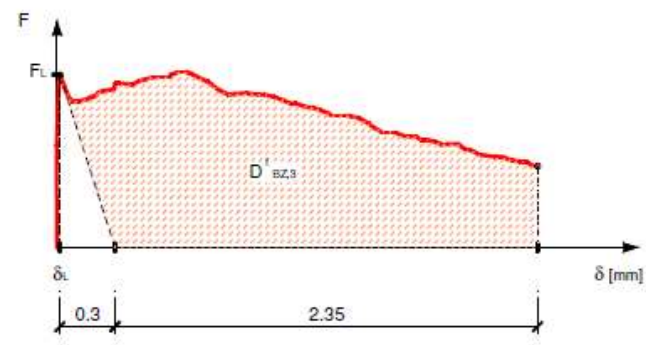

$a_{2}$ ) Avaliação de $f_{\text {eq,3 }}$

a) Curva carga $x$ deslocamento típica 


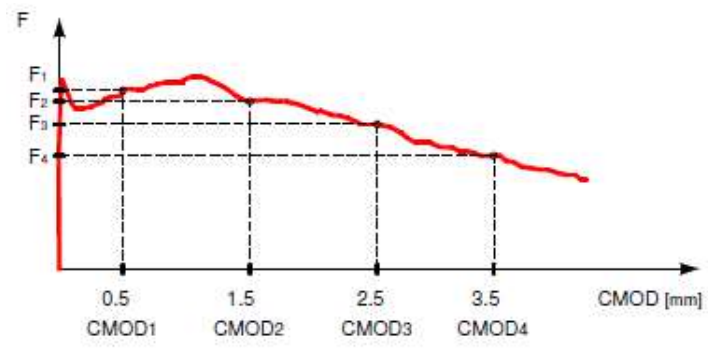

b) Curva carga $x$ CMOD típica

Figura 4: Teste de flexão sugerido pelo RILEM TC 162-TDF (2002). Fonte: RILEM TC 162-TDF (2002).

O cálculo do momento resistente teórico será estabelecido para peças de concreto com seção transversal retangular, reforçada com fibra de aço e com armadura de flexão nas faces tracionada e comprimida, conforme mostra a Figura 5. O processo será realizado iterativamente, com auxílio da ferramenta SOLVER do Excel, com intuito de determinar a altura da linha neutra.

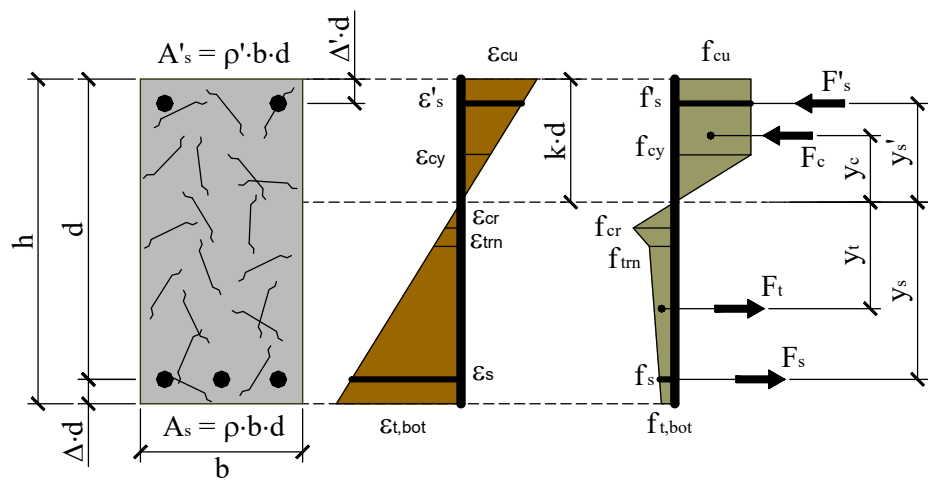

Figura 5: Distribuição das deformações e tensões. Fonte: RILEM TC 162-TDF (2002).

\section{Metodologia de Análise dos Resultados}

O método de análise de resultados tem como alvo conferir os critérios de dimensionamento exemplificado pelas normas com os resultados experimentais, medindo a confiabilidade da norma, para isso será realizado a razão entre as forças residuais experimentais (coletados no BD, tabela 1) e teóricas (estimados pelas propostas) $\lambda_{\mathrm{i}}=M_{\mathrm{Ri}, \text { exp }} / M_{\mathrm{Ri}, \text { teo, }}$ cujos valores serão apresentados nos resultados.

Quadro 3: Escala de demérito de Collins.

\begin{tabular}{|l|l|l|}
\hline $\boldsymbol{M}_{\text {Ri,exp }} / \boldsymbol{M}_{\text {Ri,calc }}$ & Escore & Classificação \\
\hline$<0,50$ & 10 & Extremamente perigosa \\
\hline $0,50 \mid----0,65$ & 5 & Perigosa \\
\hline $0,65 \mid---0,85$ & 2 & Baixa Segurança \\
\hline $0,85 \mid----1,30$ & 0 & Segurança Apropriada \\
\hline $1,30 \mid----2,00$ & 1 & Conservativa \\
\hline$\geq 2$ & 2 & Extremamente conservativa \\
\hline
\end{tabular}

Dessa forma a média demonstra um viés conservativo da equação, o coeficiente de variação é utilizado como indicador de precisão dos resultados e os menores e maiores valores complementam proporcionando a amplitude. Tendo como intuito estudar a adequação e comparação entre equações normativas de dimensionamento resistência a tração na flexão no CRFA, foi introduzido um abrangente estudo realizado por Collins, pois neste é desenvolvido um procedimento que considera distintos critérios normativos de dimensionamento e os classificam, analisando a segurança, a exatidão e a economia, ver 
Quadro 2.

\section{RESULTADOS E DISCUSSÃO}

Serão apresentados e discutidos os resultados dos Momentos Resistentes Teóricos encontrados através dos métodos do RILEM, em comparação com o Momento encontrado experimentalmente. Abaixo segue o Quadro 3 com os Momentos Resistentes. seguidos pelo coeficiente que relaciona os momentos experimentais obtidos no banco de dados e os momentos resistentes teóricos.

Quadro 4: Relação entre Momento Resistente Experimental e Teórico.

\begin{tabular}{|c|c|c|c|c|}
\hline Autor & Peça & $\begin{array}{l}M_{R, E X P} \\
\text { (kN.m) }\end{array}$ & $\begin{array}{l}M_{R, \text { teo }} \text { (RILEM) } \\
\text { (kN.m) }\end{array}$ & $\lambda_{\text {RILEM }}$ \\
\hline \multirow[t]{4}{*}{ LIMA (2012) } & Vigas 6 & 19,34 & 19,33 & 1,00 \\
\hline & Vigas 7 & 11,80 & 19,33 & 0,61 \\
\hline & Vigas 10 & 12,60 & 11,38 & 1,11 \\
\hline & Vigas 13 & 11,03 & 14,30 & 0,77 \\
\hline \multirow[t]{6}{*}{ GRIBNIAK et al. (2012) } & S3 -1 - F05 & 44,50 & 37,76 & 1,18 \\
\hline & S3 -2 - F05 & 46,00 & 37,76 & 1,22 \\
\hline & S3 -1 - F10 & 52,00 & 40,22 & 1,29 \\
\hline & S3 -2 - F10 & 52,30 & 40,22 & 1,30 \\
\hline & S3 -1 - F15 & 54,00 & 43,65 & 1,24 \\
\hline & S3 -2 - F15 & 53,60 & 43,65 & 1,23 \\
\hline FRITIH (2012) & Serie 'A' FRSCC & 37,86 & 40,21 & 0,94 \\
\hline ALTUN (2005) & S01 - 0,4 & 60,30 & 65,05 & 0,93 \\
\hline \multirow[t]{22}{*}{ BESHARA (2012) } & Serie $[6]-0,5$ & 12,58 & 14,32 & 0,88 \\
\hline & Serie [6] - 1,0 & 13,55 & 15,93 & 0,85 \\
\hline & Serie [6] - 2,0 & 14,02 & 18,52 & 0,76 \\
\hline & Serie [13] - 0,75 & 9,60 & 11,85 & 0,81 \\
\hline & Serie $[13]-1,50$ & 10,95 & 13,07 & 0,84 \\
\hline & Serie [10] - 0,50 & 4,85 & 6,48 & 0,75 \\
\hline & Serie $[10]-1,00$ & 5,00 & 6,85 & 0,73 \\
\hline & Serie $[10]-1,50$ & 5,40 & 7,15 & 0,76 \\
\hline & Serie [14] - 0,5 & 6,87 & 8,06 & 0,85 \\
\hline & Serie $[14]-1,0$ & 7,19 & 8,41 & 0,85 \\
\hline & Serie [14] - 1,5 & 7,22 & 8,70 & 0,83 \\
\hline & Serie $[14]-2,0$ & 7,51 & 8,96 & 0,84 \\
\hline & Serie $[15]-0,5$ & 61,20 & 48,17 & 1,27 \\
\hline & Serie $[15]-1,0$ & 61,50 & 51,05 & 1,20 \\
\hline & Serie [15] - 1,5 & 64,80 & 53,43 & 1,21 \\
\hline & Serie $[16]-0,5$ & 90,69 & 92,66 & 0,98 \\
\hline & Serie $[16]-1,0$ & 95,41 & 97,26 & 0,98 \\
\hline & Serie [16] - 1,5 & 100,40 & 101,91 & 0,99 \\
\hline & Serie [17] - 1,2 & 3,12 & 3,29 & 0,95 \\
\hline & Serie [17] - 1,2 & 17,40 & 16,76 & 1,04 \\
\hline & Serie [17] - 0,89 & 2,90 & 2,87 & 1,01 \\
\hline & Serie [17] - 0,89 & 17,27 & 16,22 & 1,06 \\
\hline \multirow[t]{2}{*}{ BĂRBOS (2013) } & FT 5.1-1 & 50,00 & 55,60 & 0,90 \\
\hline & FT 5.2-1 & 50,00 & 56,41 & 0,89 \\
\hline \multirow[t]{7}{*}{ KANG et al (2012) } & FHC-65-1.5 & 45,73 & 43,20 & 1,06 \\
\hline & FHC-100-1.5 & 48,93 & 44,78 & 1,09 \\
\hline & \multicolumn{4}{|c|}{ Continuação Quadro 5: Relação entre Momento experimental e Momento Teórico } \\
\hline & FNC-65-1 & 37,11 & 28,76 & 1,29 \\
\hline & FNC-100-1 & 37,86 & 29,62 & 1,28 \\
\hline & FNC-65-1.5 & 41,88 & 41,18 & 1,02 \\
\hline & FNC-100-1.5 & 43,11 & 42,06 & 1,02 \\
\hline \multirow[t]{3}{*}{ KHALIL (2013) } & D250V0,5h & 25,20 & 28,31 & 0,89 \\
\hline & D250V0,75h & 27,60 & 29,56 & 0,93 \\
\hline & D250V1,0h & 29,10 & 30,67 & 0,95 \\
\hline \multirow[t]{2}{*}{ RODRIGUEZ JR (2009) } & VIGA 1-1 & 20,70 & 17,53 & 1,18 \\
\hline & VIGA 1-2 & 25,28 & 21,42 & 1,18 \\
\hline
\end{tabular}




\begin{tabular}{|c|c|c|c|c|}
\hline & VIGA 1-3 & 27,83 & 22,36 & 1,24 \\
\hline & VIGA 2-1 & 39,00 & 29,91 & 1,30 \\
\hline & VIGA 2-2 & 46,35 & 33,17 & 1,40 \\
\hline & VIGA 2-3 & 42,00 & 33,64 & 1,25 \\
\hline \multirow[t]{6}{*}{ SWAMY et al. (1981) } & DR11 & 23,25 & 18,42 & 1,26 \\
\hline & DR12 & 23,81 & 19,36 & 1,23 \\
\hline & DR21 & 33,68 & 28,96 & 1,16 \\
\hline & DR22 & 35,06 & 30,11 & 1,16 \\
\hline & DR31 & 28,62 & 23,41 & 1,22 \\
\hline & DR32 & 30,83 & 24,36 & 1,27 \\
\hline \multirow[t]{4}{*}{ BYUNG (1992) } & S1V1 & 15,23 & 16,09 & 0,95 \\
\hline & S1V2 & 17,96 & 17,94 & 1,00 \\
\hline & S2V1 & 22,64 & 22,56 & 1,00 \\
\hline & S2V2 & 23,37 & 24,38 & 0,96 \\
\hline LOPES (2005) & V2 & 55,77 & 50,03 & 1,11 \\
\hline \multirow[t]{4}{*}{ KOVÁCS et al. (2003) } & A5 & 21,06 & 19,82 & 1,06 \\
\hline & A6 & 21,00 & 19,96 & 1,05 \\
\hline & A8 & 21,00 & 19,82 & 1,06 \\
\hline & A9 & 21,96 & 19,96 & 1,10 \\
\hline \multirow[t]{2}{*}{ NAGHIBDEHI et al. (2014) } & PP0.5 & 3,20 & 4,02 & 0,80 \\
\hline & PP1.0 & 4,68 & 6,93 & 0,67 \\
\hline
\end{tabular}

\section{Análise por método de Collins}

Analisando os valores do banco de dados através da escala de demérito de Collins, foi obtido os valores do Quadro 4, classificando a quantidade de peças com suas respectivas penalidades.

Quadro 6: Análise do BD através da escala demérito de Collins.

\begin{tabular}{|l|l|l|}
\hline Modelo & RILEM & \multicolumn{2}{l|}{} \\
\hline$\lambda=\mathrm{M}_{\mathrm{R}, \mathrm{EXP}} / \mathrm{M}_{\mathrm{R}, \text { TEO }}$ & $\mathrm{N}^{\circ}$ peça & Penal. \\
\hline$<0.50$ & 0 & 0 \\
\hline$[0.50-0.85[$ & 15 & 75 \\
\hline$[0.85-1.15[$ & 30 & 0 \\
\hline$[1.15-2.00[$ & 23 & 23 \\
\hline$\geq 2.00$ & 0 & 0 \\
\hline Total & 68 & 98 \\
\hline Resumo estatístico & & \\
\hline Modelo & $\mathrm{RILEM}$ & \\
\hline Média (Med) & 1,03 & \\
\hline DP & 0,18 & \\
\hline CV (\%) & 17,84 & \\
\hline
\end{tabular}

Apesar da média ter sido próxima de 1, o desvio padrão e o coeficiente de variação foi relativamente alto, o que acaba trazendo um resultado negativo a pesquisa. Pelo o critério de penalidade a norma apresenta 30 peças classificadas como o nível de segurança mais apropriado, sem levar penalidade alguma, entre tanto 15 peças estão na margem perigosa da escala de demérito que resulta na penalidade de 75 pontos, porém dessas 15 peças na margem perigosa 11 são do estudo de Beshara (2012), o diferencial de Beshara para os outros estudos é que ele utilizou o concreto de alto desempenho.

O gráfico a seguir mostra a relação momento experimental x momento teórico os valores analisados a seguir apresentam uma dispersão considerável ver Figura 5. No gráfico de barras (Figura 6) vemos que apenas $44 \%$ dos valores obtidos estão dentro do fator de segurança de $\lambda \geq 1$ menos da metade do ensaio total. 
RILEM

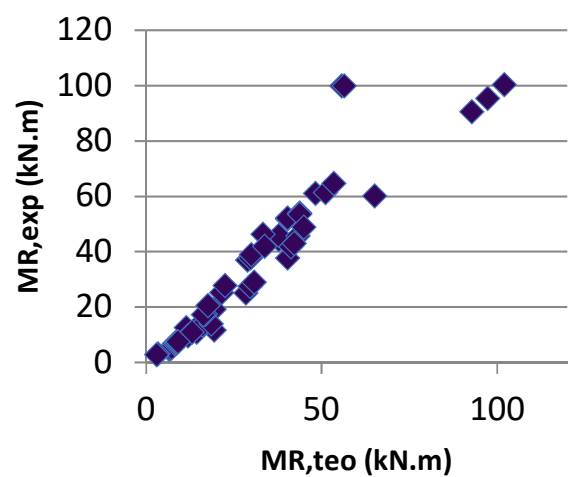

Figura 6: Gráfico da Relação entre $M R$,EXP e $M R$,TEO

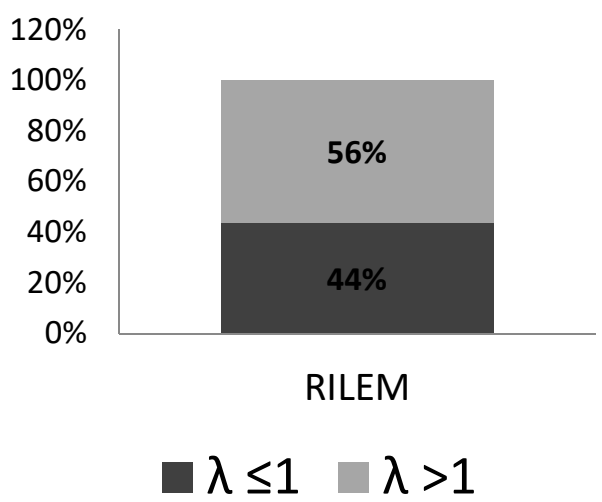

Figura 7: Gráfico da Avaliação de $\lambda$.

\section{CONCLUSÕES}

Conclui-se então que a norma RILEM TC 162-TDF (2002) possui uma confiabilidade para alguns tipos de vigas utilizando os devidos materiais. Porem quando utilizado o concreto de alto desempenho ela acaba se tornando não confiável. Pois os resultados quais apresentaram nível de segurança menor foi o de Beshara (2002), o que de modo geral acabou influenciando para um resultado insatisfatório pois das 23 amostras de ensaio 11 obtiveram valores inseguros. Seria necessária uma continuação do estudo apenas com parâmetros semelhantes de traço, para que se fosse obtido o resultado mais preciso sobre a confiabilidade da norma.

\section{REFERÊNCIAS}

BARBOS, G. A.. A spects of flexural behavior of high strength Urbanism Arhitectura Constructii, v.4, n.2, p.53-58, 2013.

BESHARA, F. B. A.; SHAABAN, I. G.; MUSTAFA, T. S.. Nominal Flexural Strength of High Strength Fiber Reinforced Concrete Beams. Arabian Journal for Science and Engineering, v.37, n.2, p.291-301, 2012.

FRITIH, Y.; VIDAL, T.; TURTSINZE, A. PONS, G.. Flexural and shear behavior of steel fiber reinforced SCC beams. KSCE Journal of Civil Engineering, v.17, n.6, p.1383-1393, 2013.

GRIBNIAK, V.; KAKLAUSKAS, G.; KWAN, A. K. H.; BACINSKAS, D.; ULBINAS, D.. Deriving stress-strain relationships for steel fibre concrete in tension from tests of beams with ordinary reinforcement. Engineering Structures, v.42, p.387-395, 2012.

GRIBNIAK, V.; KAČIANAUSKAS, R.; KAKLAUSKAS, G.; KLIUKAS, R.. Improving efficiency of inverse constitutive analysis of reinforced concrete flexural members. Scientific Research And Essays, v.7, n.8, p.923-938, 2012.

KANG, T. H. K.; MASSONE, L. M.; KIM, W.; GALLEGUILLOS, T. A.. Shear-flexure coupling behavior of steel fiber-reinforced concrete beams. ACI Structural Journal, v.109, n.4, p.435444, 2012.

KHALIL, W. I.; TAYFUR, Y. R.. Flexural strength of fibrous ultra-high performance reinforced concrete beams. ARPN Journal of Engineering and Applied Sciences, v.8, n.3, p.200214, 2013
KOVÁCS, I.; BALÁZS, G. L.. Structural behaviour of steel fibre reinforced concrete. Structural Concrete, v.4, n.2, p.57-63, 2003.

LIMA, R. P. A. F. P.. Utilização de fibras metálicas com funções estruturais em estruturas de betão. Porto: Universidade do Porto, 2012

NAGHIBDEHI, M. G.; SHARBATDAR. M.; MASTALI, M.; NAGHIBDEHI, M. G.. Flexural performance of functionally graded RC cross-section with steel and PP fibres. Magazine Of Concrete Research, v.66, n.5, p.219-233, 2014.

RILEM TECHNICAL COMMITTEE. RILEM 162 - TDF: Test and methods for steel fibre reinforced concrete, Materials and Structures. v.36. 2002.

RODRIGUES JÚNIOR, A.. Comportamento de vigas de concreto com fibras e baixas taxas de armadura longitudinal. Rio de Janeiro: UFRJ, 2009.

SWAMY, R. N.; AL-TA'AN, S. A.. Deformation and Ultimate Strength in Flexure of Reinforced Concrete Beams Made With Steel Fiber Concrete. Journal of the American Concrete Institute, v.78, n.5, p.395-405, 1981.

VELASCO, R. V.. Concretos Auto Adensáveis Reforçados com Elevadas Frações Volumétricas de Fibras de Aço: Propriedades Reológicas, Físicas, Mecânicas e Térmicas. 2009.

A CBPC - Companhia Brasileira de Produção Científica (CNPJ: 11.221.422/0001-03) detém os direitos materiais desta publicação. Os direitos referem-se à publicação do trabalho em qualquer parte do mundo, incluindo os direitos às renovações, expansões e disseminações da contribuição, bem como outros direitos subsidiários. Todos os trabalhos publicados eletronicamente poderão posteriormente ser publicados em coletâneas impressas sob coordenação da Sustenere Publishing, da Companhia Brasileira de Produção Científica e seus parceiros autorizados. Os (as) autores (as) preservam os direitos autorais, mas não têm permissão para a publicação da contribuição em outro meio, impresso ou digital, em português ou em tradução. 